\title{
Nontrivial homoclinic solutions for prescribed mean curvature Rayleigh equations
}

Meilin Zheng ${ }^{1}$ and Jin $\mathrm{Li}^{2^{*}}$

\section{${ }^{\text {*Correspondence: }}$} lijin7912@gmail.com ${ }^{2}$ School of Science, Jiujiang University, Jiujiang, 332005, China Full list of author information is available at the end of the article

\begin{abstract}
In this paper, some sufficient conditions on the existence of $2 k \pi$-periodic solutions for a kind of prescribed mean curvature Rayleigh equations are given. Then the existence of nontrivial homoclinic solutions for prescribed mean curvature Rayleigh equations is obtained.
\end{abstract}

Keywords: prescribed mean curvature Rayleigh equation; homoclinic solution; Mawhin's continuation theorem

\section{Introduction}

In this paper, we are concerned with the existence of homoclinic solutions for the following prescribed mean curvature Rayleigh equation:

$$
\left(\frac{x^{\prime}(t)}{\sqrt{1+x^{\prime 2}(t)}}\right)^{\prime}+f\left(x^{\prime}(t)\right)+g(x(t))=e(t)
$$

where $f, g, e \in C(\mathbb{R}, \mathbb{R})$ with $f(0)=0$ and $g(0)=0$.

A solution $x(t)$ of (1.1) is named homoclinic (to 0 ) if $x(t) \rightarrow 0$ and $x^{\prime}(t) \rightarrow 0$ as $|t| \rightarrow 0$. Furthermore, $x(t)$ is called a nontrivial homoclinic solution (1.1) if $x(t)$ is a homoclinic solution of (1.1) and $x \neq 0$.

Various types of prescribed mean equations have been studied widely by some authors in many papers (see [1-8]) because of their having appeared in some scientific fields, such as differential geometry and physics. Recently, some results on the existence of solutions for prescribed mean equations were obtained (see [9-12] and references cited therein). Feng in [10] discussed a delay prescribed mean curvature Liénard equation of the form

$$
\left(\frac{x^{\prime}(t)}{\sqrt{1+x^{\prime 2}(t)}}\right)^{\prime}+f(x(t)) x^{\prime}(t)+g(t, x(t-\tau(t)))=e(t)
$$

estimated a priori bounds by eliminating the nonlinear term $\left(\frac{x^{\prime}(t)}{\sqrt{1+x^{\prime 2}(t)}}\right)^{\prime}$ and established sufficient conditions on the existence of periodic solutions for (1.2) by using Mawhin's continuation theorem. The main difficulty overcome by Feng in [10] lies in the nonlinear term $\left(\frac{x^{\prime}(t)}{\sqrt{1+x^{\prime 2}(t)}}\right)^{\prime}$, the existence of which obstructs the usual method of finding a priori

\section{Springer}

(c) 2015 Zheng and Li; licensee Springer. This is an Open Access article distributed under the terms of the Creative Commons Attribution License (http://creativecommons.org/licenses/by/4.0), which permits unrestricted use, distribution, and reproduction in any medium, provided the original work is properly credited. 
bounds. He transformed (1.2) into the following equivalent system:

$$
\left\{\begin{array}{l}
x_{1}^{\prime}(t)=\psi\left(x_{2}(t)\right)=\frac{x_{2}(t)}{\sqrt{1-x_{2}^{2}(t)}} \\
x_{2}^{\prime}(t)=-f\left(x_{1}(t)\right) \psi\left(x_{2}(t)\right)-g\left(t, x_{1}(t)\right)+e(t)
\end{array}\right.
$$

and used Mawhin's continuation theorem to prove the existence result. From then on, similar approaches were used by Li and Wang [13] and Li et al. [14]. Clearly, $\left|x_{2}\right|<c<1$ ( $c$ is a constant) is necessarily satisfied when Mawhin's continuation theorem is applied to the system (1.3). But, as pointed out by Liang and Lu in [15], the proof of $\left|x_{2}\right|<c<1$ was not done in $[10,13,14]$. To do it, Li and Wang in [16] gave a complementary proof.

In [15], Liang and Lu investigated the following prescribed mean curvature Duffing equation:

$$
\left(\frac{x^{\prime}(t)}{\sqrt{1+x^{\prime 2}(t)}}\right)^{\prime}+c x^{\prime}(t)+f(x(t))=p(t)
$$

where $f \in C^{1}(\mathbb{R}, \mathbb{R}), c>0$. Assume

$\left(\mathrm{A}_{1}\right)$ there exist constants $m_{0}>0, \alpha>1$ such that $x f(x) \leq-m_{0}|x|^{\alpha}$ and $f^{\prime}(x)<0, \forall x \in \mathbb{R}$,

and

$\left(\mathrm{A}_{2}\right) p \in C(\mathbb{R}, \mathbb{R})$ is a bounded function with $p(t) \neq 0$ and

$$
B:=\max \left\{\left(\int_{\mathbb{R}}|p(t)|^{2} d t\right)^{\frac{1}{2}},\left(\int_{\mathbb{R}}|p(t)|^{\beta} d t\right)^{\frac{1}{\beta}}\right\}+\sup _{t \in \mathbb{R}}|p(t)|<+\infty
$$

where $\frac{1}{\alpha}+\frac{1}{\beta}=1$.

If $\left(\mathrm{A}_{1}\right),\left(\mathrm{A}_{2}\right)$, and condition

$$
B^{\frac{\alpha}{2 \alpha-2}}+T B m_{0}^{\frac{1}{2 \alpha-2}}<\sqrt{2 T} m_{0}^{\frac{1}{2 \alpha-2}}
$$

hold, they obtained the existence of homoclinic solutions for (1.4). For the method of obtaining homoclinic solution, we also see the papers [17-20]. It is not difficult to see that the condition (1.5) is complex and strong. In this paper, we will consider more general equation (1.1) and obtain the existence of homoclinic solutions under the more simple and reasonable conditions. To find a homoclinic solution for (1.1), we seek a limit of a certain sequence of $2 k T$-periodic solutions $x_{k}(t)$ for the following equations:

$$
\left(\frac{x^{\prime}(t)}{\sqrt{1+x^{\prime 2}(t)}}\right)^{\prime}+f\left(x^{\prime}(t)\right)+g(x(t))=e_{k}(t)
$$

where $k \in \mathbb{N}, e_{k}: \mathbb{R} \rightarrow \mathbb{R}$ is a $2 k T$-periodic function ( $T>0$ is a constant) defined by

$$
e_{k}(t)= \begin{cases}e(t), & t \in\left[-k T, k T-\varepsilon_{0}\right), \\ e\left(k T-\varepsilon_{0}\right)+\frac{e(-k T)-e\left(k T-\varepsilon_{0}\right)}{\varepsilon_{0}}\left(t-k T+\varepsilon_{0}\right), & t \in\left[k T-\varepsilon_{0}, k T\right],\end{cases}
$$

$\varepsilon_{0} \in(0, T)$ is a constant independent of $k$. 


\section{Preliminaries}

Let $X$ and $Y$ be real Banach spaces and $L: X \supset \operatorname{Dom} L \rightarrow Y$ be a linear operator. $L$ is said to be a Fredholm operator with index zero provided that

(i) $\operatorname{Im} L$ is closed subset of $Y$,

(ii) $\operatorname{dim} \operatorname{ker} L=\operatorname{codim} \operatorname{Im} L<+\infty$.

Set $X=\operatorname{ker} L \oplus X_{1}, Y=\operatorname{Im} L \oplus Y_{1}$. Let $P: X \rightarrow \operatorname{ker} L$ and $Q: Y \rightarrow Y_{1}$ be the nature projections. It is easy to see that $\operatorname{ker} L \cap\left(\operatorname{Dom} L \cap X_{1}\right)=0$. Thus the restriction $L_{P}:=\left.L\right|_{\operatorname{Dom} L \cap X_{1}}$ is invertible. We denote by $K$ the inverse of $L_{P}$.

Let $\Omega$ be an open bounded subset of $X$ with $\operatorname{Dom} L \cap \Omega \neq \phi$. A map $N: \bar{\Omega} \rightarrow Y$ is said to be $L$-compact in $\bar{\Omega}$ if $Q N: \bar{\Omega} \rightarrow Y$ and $K(I-Q) N: \bar{\Omega} \rightarrow X$ are compact.

The following lemma due to Mawhin (see [21]) is a fundamental tool to prove the existence of $2 k T$-periodic solutions for (1.6).

Lemma 2.1 Let $L$ be a Fredholm operator of index zero and Let $N$ be L-compact on $\bar{\Omega}$. If the following conditions hold.

$\left(\mathrm{h}_{1}\right) L x \neq \lambda N x, \forall(x, \lambda) \in[(D(L) \backslash \operatorname{ker} L) \cap \partial \Omega] \times(0,1)$;

$\left(\mathrm{h}_{2}\right) N x \notin \operatorname{Im} L, \forall x \in \operatorname{ker} L \cap \partial \Omega$;

$\left(\mathrm{h}_{3}\right) \operatorname{deg}\left(\left.J Q N\right|_{\mathrm{ker} L}, \Omega \cap \operatorname{ker} L, 0\right) \neq 0$, where $J: \operatorname{Im} Q \rightarrow \operatorname{ker} L$ is an isomorphism.

Then $L x=N x$ has at least one solution in $D(L) \cap \bar{\Omega}$.

The following lemma is a special case of Lemma 2.1 in [22].

Lemma 2.2 If $x: \mathbb{R} \rightarrow \mathbb{R}$ is continuously differentiable on $\mathbb{R}, a>0$, then the following inequality holds:

$$
|x(t)| \leq(2 a)^{-\frac{1}{2}}\left(\int_{t-a}^{t+a}|x(s)|^{2} d s\right)^{\frac{1}{2}}+a(2 a)^{-\frac{1}{p}}\left(\int_{t-a}^{t+a}\left|x^{\prime}(s)\right|^{2} d s\right)^{\frac{1}{2}} .
$$

Lemma 2.3 [22] Let $x_{k} \in C_{2 k T}^{2}$ be $2 k T$-periodic function for each $k \in \mathbb{N}$ with

$$
\left\|x_{k}\right\|_{\infty} \leq A_{0}, \quad\left\|x_{k}^{\prime}\right\|_{\infty} \leq A_{1}, \quad\left\|x_{k}^{\prime \prime}\right\|_{\infty} \leq A_{2}
$$

where $A_{0}, A_{1}$, and $A_{2}$ are constants independent of $k \in \mathbb{N}$. Then there exists a function $x_{0} \in C^{1}(\mathbb{R}, \mathbb{R})$ such that for each interval $[c, d] \subset \mathbb{R}$, there is a subsequence $\left\{x_{k_{j}}\right\}$ of $\left\{x_{k}\right\}_{k \in \mathbb{N}}$ with $x_{k_{j}}^{\prime}(t) \rightarrow x_{0}^{\prime}(t)$ uniformly on $[c, d]$.

In order to apply Mawhin's continuation theorem to study the existence of $2 k T$-periodic solution of (1.6), we rewrite (1.6) as

$$
\left\{\begin{array}{l}
x^{\prime}(t)=\frac{y(t)}{\sqrt{1-y^{2}(t)}} \\
y^{\prime}(t)=-f\left(\frac{y(t)}{\sqrt{1-y^{2}(t)}}\right)-g(x(t))+e_{k}(t)
\end{array}\right.
$$

Obviously, if $z(t)=(x(t), y(t))^{\top}$ is a $2 k T$-periodic solution of (2.2), then $x(t)$ must be a $2 k T$-periodic solution of (1.6). Hence, the problem of finding a $2 k T$-periodic solution of (1.6) reduces to finding one of (2.2). 
Now, we set

$$
X_{k}=Y_{k}=\left\{z: z(t)=(x(t), y(t))^{\top} \in C\left(\mathbb{R}^{1}, \mathbb{R}^{2}\right), z(t)=z(t+2 k T)\right\}
$$

with the norm $\|z\|=\max \left\{\|x\|_{\infty},\|y\|_{\infty}\right\}$, where

$$
\|x\|_{\infty}=\max _{t \in[0,2 k T]}|x(t)|, \quad\|y\|_{\infty}=\max _{t \in[0,2 k T]}|y(t)| .
$$

Clearly, $X_{k}$ and $Y_{k}$ are Banach spaces. Meanwhile, let

$$
L: X_{k} \supset \operatorname{Dom} L \rightarrow Y_{k}, \quad L z=z^{\prime}=\left(x^{\prime}(t), y^{\prime}(t)\right)^{\top},
$$

where

$$
\operatorname{Dom} L=\left\{z: z=(x(t), y(t))^{\top} \in C^{1}\left(\mathbb{R}, \mathbb{R}^{2}\right), z(t)=z(t+2 k T)\right\} .
$$

Define a nonlinear operator $N: X_{k} \rightarrow Y_{k}$ by

$$
N z=\left(\begin{array}{c}
\frac{y(t)}{\sqrt{1-y^{2}(t)}} \\
-f\left(\frac{y(t)}{\sqrt{1-y^{2}(t)}}\right)-g(x(t))+e(t)
\end{array}\right) .
$$

Then the system (2.2) can be written to $L z=N z$.

It is easy to see that $\operatorname{ker} L=\mathbb{R}^{2}$ and $\operatorname{Im} L=\left\{u \in Y_{k}: \int_{-k T}^{k T} u(s) d s=0\right\}$. So $L$ is a Fredholm operator with index zero.

Let $P: X_{k} \rightarrow \operatorname{ker} L$ and $Q: Y_{k} \rightarrow \operatorname{Im} Q$ be defined by

$$
P z=\frac{1}{2 k T} \int_{-k T}^{k T} z(s) d s, \quad Q u=\frac{1}{2 k T} \int_{-k T}^{k T} u(s) d s
$$

and denote by $K$ the inverse of $\left.L\right|_{\operatorname{ker} P \cap \operatorname{Dom} L}$. Then, $\operatorname{ker} L=\operatorname{Im} Q=\mathbb{R}^{2}$ and

$$
K u(t)=\int_{-k T}^{k T} G(t, s) u(s) d s
$$

where

$$
G(t, s)= \begin{cases}\frac{s}{2 k T}, & -k T \leq s<t \leq k T \\ \frac{s-k T}{2 k T}, & -k T \leq t \leq s \leq k T .\end{cases}
$$

It follows from (2.3) that $N$ is $L$-compact on $\bar{\Omega}$, where $\Omega$ is an open, bounded subset of $X_{k}$.

\section{Main result}

For the sake of convenience, we give the following fundamental assumptions.

$\left(\mathrm{H}_{1}\right)$ There exist $\alpha$ and $\beta$ with $\beta>\alpha>0$ such that $\forall x \in \mathbb{R}$,

$$
\alpha x^{2} \leq x f(x) \leq \beta x^{2}
$$


$\left(\mathrm{H}_{2}\right)$ There exists $\gamma>0$ such that

$$
x g(x) \leq-\gamma x^{2}
$$

$\left(\mathrm{H}_{3}\right) e(t)$ is bounded on $\mathbb{R}$ and

$$
0<d:=\max \left\{\left(\int_{-\infty}^{+\infty} e^{2}(t) d t\right)^{\frac{1}{2}}, \sup _{t \in \mathbb{R}}|e(t)|\right\}<+\infty
$$

Obviously, the conditions $\left(\mathrm{H}_{1}\right),\left(\mathrm{H}_{2}\right)$, and $\left(\mathrm{H}_{3}\right)$ are simplistic and reasonable.

Theorem 3.1 Let $\left(\mathrm{H}_{1}\right),\left(\mathrm{H}_{2}\right)$, and $\left(\mathrm{H}_{3}\right)$ hold. Then for each $k \in \mathbb{N}$, (1.6) has at least one $2 k T$-periodic solution.

Proof We consider the auxiliary system of the system (2.2),

$$
\left\{\begin{array}{l}
x^{\prime}(t)=\lambda \frac{y(t)}{\sqrt{1-y^{2}(t)}}=\lambda \psi(y(t)) \\
y^{\prime}(t)=-\lambda f(\psi(y(t)))-\lambda g(x(t))+\lambda e_{k}(t)
\end{array}\right.
$$

where $\lambda \in(0,1]$ is a parameter. Firstly, we will prove that the set of all possible $2 k T$-periodic solutions of the system (3.1) is bounded.

Obviously, the system (3.1) is equivalent to the following equation:

$$
\left(\frac{\frac{1}{\lambda} x^{\prime}(t)}{\sqrt{1+\frac{1}{\lambda^{2}} x^{\prime 2}(t)}}\right)^{\prime}+\lambda f\left(\frac{1}{\lambda} x^{\prime}(t)\right)+\lambda g(x(t))=\lambda e_{k}(t) .
$$

Multiplying (3.2) by $x^{\prime}$ and integrating from $-k T$ to $k T$, we have

$$
\lambda \int_{-k T}^{k T} f\left(\frac{1}{\lambda} x^{\prime}(t)\right) x^{\prime}(t) d t+\lambda \int_{-k T}^{k T} g(x(t)) x^{\prime}(t) d t=\lambda \int_{-k T}^{k T} e_{k}(t) x^{\prime}(t) d t
$$

By $\left(\mathrm{H}_{1}\right)$, we obtain

$$
\begin{aligned}
\left|\lambda \int_{-k T}^{k T} f\left(\frac{1}{\lambda} x^{\prime}(t)\right) x^{\prime}(t) d t\right| & \geq \lambda^{2} \int_{-k T}^{k T} f\left(\frac{1}{\lambda} x^{\prime}(t)\right) \frac{x^{\prime}(t)}{\lambda} d t \\
& \geq \alpha \int_{-k T}^{k T}\left|x^{\prime}(t)\right|^{2} d t .
\end{aligned}
$$

By (1.7), we get

$$
\begin{aligned}
\int_{-k T}^{k T}\left|e_{k}(t)\right|^{2} d t= & \int_{-k T}^{k T-\varepsilon_{0}}|e(t)|^{2} d t+\int_{k T-\varepsilon_{0}}^{k T} \mid e\left(k T-\varepsilon_{0}\right) \\
& +\left.\frac{e(-k T)-e\left(k T-\varepsilon_{0}\right)}{\varepsilon_{0}} \cdot\left(t-k T+\varepsilon_{0}\right)\right|^{2} d t \\
\leq & \int_{-k T}^{k T-\varepsilon_{0}}|e(t)|^{2} d t+\int_{k T-\varepsilon_{0}}^{k T} \max \left\{\left|e\left(k T-\varepsilon_{0}\right)\right|^{2},|e(-k T)|^{2}\right\} d t
\end{aligned}
$$




$$
\begin{aligned}
& \leq \int_{-\infty}^{+\infty}|e(t)|^{2} d t+\int_{k T-\varepsilon_{0}}^{k T} d^{2} d t \\
& \leq d^{2}\left(1+\varepsilon_{0}\right) .
\end{aligned}
$$

Noticing that $\int_{-k T}^{k T} g(x(t)) x^{\prime}(t) d t=0$, we have from (3.3), (3.4), and (3.5)

$$
\begin{aligned}
\alpha \int_{-k T}^{k T}\left|x^{\prime}(t)\right|^{2} d t & \leq\left|\lambda \int_{-k T}^{k T} f\left(\frac{1}{\lambda} x^{\prime}(t)\right) x^{\prime}(t) d t\right| \\
& \leq \lambda\left(\left|\int_{-k T}^{k T} g(x(t)) x^{\prime}(t) d t\right|+\left|\int_{-k T}^{k T} e_{k}(t) x^{\prime}(t) d t\right|\right) \\
& \leq \lambda\left(\int_{-k T}^{k T} e_{k}^{2}(t) d t\right)^{\frac{1}{2}} \cdot\left(\int_{-k T}^{k T}\left|x^{\prime}(t)\right|^{2} d t\right)^{\frac{1}{2}} \\
& \leq \lambda d \sqrt{1+\varepsilon_{0}} \cdot\left(\int_{-k T}^{k T}\left|x^{\prime}(t)\right|^{2} d t\right)^{\frac{1}{2}},
\end{aligned}
$$

i.e.,

$$
\left(\int_{-k T}^{k T}\left|x^{\prime}(t)\right|^{2} d t\right)^{\frac{1}{2}} \leq \frac{\lambda d}{\alpha} \sqrt{1+\varepsilon_{0}}
$$

Hence, there exists a positive constant $D_{1}$ independent of $k$ and $\lambda$ such that

$$
\left\|x^{\prime}\right\|_{2} \leq D_{1}
$$

Multiplying (3.2) by $x$ and integrating from $-k T$ to $k T$, we obtain

$$
\begin{aligned}
& \int_{-k T}^{k T}\left(\frac{\frac{1}{\lambda} x^{\prime}(t)}{\sqrt{1+\frac{1}{\lambda^{2}} x^{\prime 2}(t)}}\right)^{\prime} x(t) d t+\lambda \int_{-k T}^{k T} f\left(\frac{1}{\lambda} x^{\prime}(t)\right) x(t) d t+\lambda \int_{-k T}^{k T} g(x(t)) x(t) d t \\
& \quad=\lambda \int_{-k T}^{k T} e_{k}(t) x(t) d t .
\end{aligned}
$$

Since $x^{\prime}(t)=\lambda \frac{y(t)}{\sqrt{1-y^{2}(t)}}$, we get $y(t)=\frac{\frac{1}{\lambda} x^{\prime}(t)}{\sqrt{1+\frac{1}{\lambda^{2}} x^{\prime 2}(t)}}$. Then

$$
\begin{aligned}
\int_{-k T}^{k T}\left(\frac{\frac{1}{\lambda} x^{\prime}(t)}{\sqrt{1+\frac{1}{\lambda^{2}} x^{\prime 2}(t)}}\right)^{\prime} x(t) d t & =\int_{-k T}^{k T} y^{\prime}(t) x(t) d t \\
& =-\int_{-k T}^{k T} y(t) x^{\prime}(t) d t \\
& =-\lambda \int_{-k T}^{k T} \frac{y^{2}(t)}{\sqrt{1-y^{2}(t)}} d t .
\end{aligned}
$$

It follows from (3.8) and (3.9) that

$$
-\int_{-k T}^{k T} g(x(t)) x(t) d t \leq-\int_{-k T}^{k T} f\left(\frac{1}{\lambda} x^{\prime}(t)\right) x(t) d t+\int_{-k T}^{k T} e_{k}(t) x(t) d t .
$$


By $\left(\mathrm{H}_{2}\right)$, we have

$$
\int_{-k T}^{k T} g(x(t)) x(t) d t \leq-\int_{-k T}^{k T} \gamma x^{2}(t) d t=-\gamma\|x\|_{2}^{2} .
$$

By $\left(\mathrm{H}_{1}\right)$ and (3.6), we obtain

$$
\begin{aligned}
-\int_{-k T}^{k T} f\left(\frac{1}{\lambda} x^{\prime}(t)\right) x(t) d t & \leq \beta \int_{-k T}^{k T}\left|\frac{1}{\lambda} x^{\prime}(t)\right||x(t)| d t \\
& \leq \beta\left(\int_{-k T}^{k T}\left|\frac{1}{\lambda} x^{\prime}(t)\right|^{2} d t\right)^{\frac{1}{2}} \cdot\left(\int_{-k T}^{k T}|x(t)|^{2} d t\right)^{\frac{1}{2}} \\
& \leq \frac{\beta}{\lambda} \cdot\left\|x^{\prime}\right\|_{2} \cdot\|x\|_{2} \\
& \leq \frac{\beta d}{\alpha} \sqrt{1+\varepsilon_{0}}\|x\|_{2} .
\end{aligned}
$$

By (3.5), we get

$$
\begin{aligned}
\int_{-k T}^{k T} e_{k}(t) x(t) d t & \leq\left(\int_{-k T}^{k T} e_{k}^{2}(t) d t\right)^{\frac{1}{2}} \cdot\left(\int_{-k T}^{k T}|x(t)|^{2} d t\right)^{\frac{1}{2}} \\
& \leq d \sqrt{1+\varepsilon_{0}}\|x\|_{2} .
\end{aligned}
$$

Then we get from (3.10), (3.11), (3.12), and (3.13)

$$
\gamma\|x\|_{2}^{2} \leq \frac{\beta d}{\alpha} \sqrt{1+\varepsilon_{0}}\|x\|_{2}+d \sqrt{1+\varepsilon_{0}}\|x\|_{2},
$$

i.e.,

$$
\|x\|_{2} \leq \frac{\alpha+\beta}{\alpha \gamma} d \sqrt{1+\varepsilon_{0}} .
$$

Therefore, there exists a positive constant $D_{2}$ independent of $k$ and $\lambda$ such that

$$
\|x\|_{2} \leq D_{2}
$$

Thus, by using Lemma 2.2, we have

$$
\begin{aligned}
|x(t)| & \leq(2 T)^{-\frac{1}{2}}\left(\int_{t-T}^{t+T}|x(s)|^{2}\right)^{\frac{1}{2}}+T(2 T)^{-\frac{1}{2}}\left(\int_{t-T}^{t+T}\left|x^{\prime}(s)\right|^{2}\right)^{\frac{1}{2}} \\
& \leq(2 T)^{-\frac{1}{2}}\left(\int_{t-k T}^{t+k T}|x(s)|^{2}\right)^{\frac{1}{2}}+T(2 T)^{-\frac{1}{2}}\left(\int_{t-k T}^{t+k T}\left|x^{\prime}(s)\right|^{2}\right)^{\frac{1}{2}} \\
& =(2 T)^{-\frac{1}{2}}\left(\int_{-k T}^{k T}|x(s)|^{2}\right)^{\frac{1}{2}}+T(2 T)^{-\frac{1}{2}}\left(\int_{-k T}^{k T}\left|x^{\prime}(s)\right|^{2}\right)^{\frac{1}{2}} \\
& \leq(2 T)^{-\frac{1}{2}} D_{2}+T(2 T)^{-\frac{1}{2}} D_{1} .
\end{aligned}
$$


Hence, there exists a positive constant $M_{1}$ independent of $k$ and $\lambda$ such that

$$
\|x\|_{\infty} \leq M_{1} .
$$

In what follows, we prove that there exists a constant $\varepsilon_{1}$ with $0<\varepsilon_{1} \ll 1$ such that

$$
|y(t)|<1-\varepsilon_{1}, \quad \forall t \in \mathbb{R} .
$$

Since $\|x\|_{\infty} \leq M_{1}$ and $g$ is continuous, there exists $M_{2}>0$ such that

$$
-M_{2}<-g(x(t))+e_{k}(t)<M_{2}, \quad \forall t \in \mathbb{R} .
$$

By $\left(\mathrm{H}_{1}\right)$, we get

$$
f(x) \geq \alpha x, \quad \forall x>0 .
$$

Now we prove by contradiction that

$$
y(t) \leq \frac{M_{2}}{\sqrt{M_{2}^{2}+\alpha^{2}}}, \quad \forall t \in \mathbb{R} .
$$

Assume that there exist $t_{2}^{*}>t_{1}^{*}$ such that

$$
y\left(t_{1}^{*}\right)=\frac{M_{2}}{\sqrt{M_{2}^{2}+\alpha^{2}}}, \quad y\left(t_{2}^{*}\right)>\frac{M_{2}}{\sqrt{M_{2}^{2}+\alpha^{2}}}
$$

and

$$
y(t)>\frac{M_{2}}{\sqrt{M_{2}^{2}+\alpha^{2}}}, \quad \forall t \in\left(t_{1}^{*}, t_{2}^{*}\right) .
$$

Noticing that $\lambda \in(0,1]$, we have $\forall t \in\left(t_{1}^{*}, t_{2}^{*}\right)$,

$$
y^{\prime}(t)=\lambda\left(-f(\psi(y(t)))-g(x(t))+e_{k}(t)\right)<0,
$$

which is a contradiction. By $\left(\mathrm{H}_{1}\right)$, we get

$$
f(x) \leq \alpha x, \quad \forall x<0 .
$$

By using a similar argument, we can prove that

$$
y(t) \geq-\frac{M_{2}}{\sqrt{M_{2}^{2}+\alpha^{2}}}, \quad \forall t \in \mathbb{R} .
$$

Thus

$$
-\frac{M_{2}}{\sqrt{M_{2}^{2}+\alpha^{2}}} \leq y(t) \leq \frac{M_{2}}{\sqrt{M_{2}^{2}+\alpha^{2}}}, \quad \forall t \in \mathbb{R} .
$$

Therefore, (3.15) holds. 
Putting

$$
\Omega=\left\{(x(t), y(t)) \in C\left(\mathbb{R}^{1}, \mathbb{R}^{2}\right):\|x\|_{\infty}<M_{1}+1,\|y\|_{\infty}<1-\varepsilon_{1}\right\}
$$

we can give a standard argument on $\Omega$ by using Lemma 2.1 (also see [13] or [14]) and find that the system (2.2) has at least one $2 k T$-periodic solution. Equivalently, (1.6) has at least one $2 k T$-periodic solution.

Theorem 3.2 Let $\left(\mathrm{H}_{1}\right),\left(\mathrm{H}_{2}\right)$, and $\left(\mathrm{H}_{3}\right)$ hold. Then (1.1) has at least one nontrivial homoclinic solution.

Proof If $\left(x_{k}(t), y_{k}(t)\right)$ is a solution of (1.6), then

$$
\left(\frac{x_{k}^{\prime}(t)}{\sqrt{1+\left(x_{k}^{\prime}(t)\right)^{2}}}\right)^{\prime}+f\left(x_{k}^{\prime}(t)\right)+g\left(x_{k}(t)\right)=e_{k}(t) .
$$

From Theorem 3.1, we have

$$
\left\|x_{k}\right\|_{\infty}<M_{1}+1, \quad\left\|y_{k}\right\|_{\infty}<1-\varepsilon_{1},
$$

where $y_{k}(t)=\frac{x_{k}^{\prime}(t)}{\sqrt{1+\left(x_{k}^{\prime}(t)\right)^{2}}}$. Equation (3.16) is equivalent to

$$
\left\{\begin{aligned}
x_{k}^{\prime}(t) & =\frac{y_{k}(t)}{\sqrt{1-y_{k}^{2}(t)}} \\
y_{k}^{\prime}(t) & =-f\left(\frac{y_{k}(t)}{\sqrt{1-y_{k}^{2}(t)}}\right)-g\left(x_{k}(t)\right)+e_{k}(t) .
\end{aligned}\right.
$$

Since $f, g, e_{k}$ are continuous, we have $y_{k}(t)$ is continuous differentiable on $\mathbb{R}$. Moreover, it follows that $x_{k}^{\prime}(t)$ is also continuous differentiable on $\mathbb{R}$. Hence,

$$
x_{k}^{\prime \prime}(t)=\frac{y_{k}^{\prime}(t)}{\left(1-y_{k}^{2}(t)\right)^{\frac{3}{2}}} .
$$

Since $f, g, e_{k}$ are continuous, we find from (3.17) that there exists a positive constant $M_{3}$ independent of $k$ such that

$$
\left\|y_{k}^{\prime}\right\|_{\infty}<M_{3}
$$

Hence, by (3.18), there exists a positive constant $M_{4}$ independent of $k$ such that

$$
\left\|x_{k}^{\prime \prime}\right\|_{\infty}<M_{4}
$$

By using Lemma 2.3, we find that there is a function $x_{0} \in C^{1}(\mathbb{R}, \mathbb{R})$ such that, for each interval $[a, b] \subset \mathbb{R}$, there is a subsequence $\left\{x_{k_{j}}(t)\right\}$ of $x_{k}(t)$ with $x_{k_{j}}^{\prime}(t) \rightarrow x_{0}^{\prime}(t)$ uniformly on $[a, b]$. In what follows, we will show that $x_{0}(t)$ is just a homoclinic solution of (1.1).

For all $a, b \in \mathbb{R}$ with $a<b$, there exist a positive integer $j_{0}$ and a positive real number $\varepsilon_{2}$ such that, for $j>j_{0},\left[a-\varepsilon_{2}, b+\varepsilon_{2}\right] \subset\left[-k_{j} T, k_{j} T-\varepsilon_{0}\right]$. Then for $j>j_{0}$,

$$
\left(\frac{x_{k_{j}}^{\prime}(t)}{\sqrt{1+\left(x_{k_{j}}^{\prime}(t)\right)^{2}}}\right)^{\prime}+f\left(x_{k_{j}}^{\prime}(t)\right)+g\left(x_{k_{j}}(t)\right)=e(t) .
$$


Since $x_{k_{j}}^{\prime}(t) \rightarrow x_{0}^{\prime}(t)$ uniformly on $[a, b]$, we have

$$
-f\left(x_{k_{j}}^{\prime}(t)\right)-g\left(x_{k_{j}}(t)\right)+e(t) \rightarrow-f\left(x_{0}^{\prime}(t)\right)-g\left(x_{0}(t)\right)+e(t), \quad \text { uniformly on }[a, b]
$$

Since $\frac{x_{k_{j}}^{\prime}(t)}{\sqrt{1+\left(x_{k_{j}}^{\prime}(t)\right)^{2}}} \rightarrow \frac{x_{0}^{\prime}(t)}{\sqrt{1+\left(x_{0}^{\prime}(t)\right)^{2}}}$ uniformly on $[a, b]$ and $\frac{x_{k_{j}}^{\prime}(t)}{\sqrt{1+\left(x_{k_{j}}^{\prime}(t)\right)^{2}}}=y_{k_{j}}(t)$ is continuous differentiable for $t \in[a, b]$, we get

$$
\left(\frac{x_{k_{j}}^{\prime}(t)}{\sqrt{1+\left(x_{k_{j}}^{\prime}(t)\right)^{2}}}\right)^{\prime} \rightarrow\left(\frac{x_{0}^{\prime}(t)}{\sqrt{1+\left(x_{0}^{\prime}(t)\right)^{2}}}\right)^{\prime}, \quad \text { uniformly on }[a, b] .
$$

Hence, we obtain

$$
\left(\frac{x_{0}^{\prime}(t)}{\sqrt{1+\left(x_{0}^{\prime}(t)\right)^{2}}}\right)^{\prime}+f\left(x_{0}^{\prime}(t)\right)+g\left(x_{0}(t)\right)=e(t), \quad t \in[a, b]
$$

Noticing that $a, b$ are two arbitrary constants with $a<b$, we find that $x_{0}: \mathbb{R} \rightarrow \mathbb{R}$ is a solution of (1.1).

In the following, we prove that $x_{0}(t) \rightarrow 0$ and $x_{0}^{\prime}(t) \rightarrow 0$ as $|t| \rightarrow+\infty$. Firstly, we prove $x_{0}(t) \rightarrow 0$ as $|t| \rightarrow+\infty$. Let $i$ be a positive integer with $i<k_{j}$, then

$$
\begin{aligned}
\int_{-i T}^{i T}\left(\left|x_{0}(t)\right|^{2}+\left|x_{0}^{\prime}(t)\right|^{2}\right) d t & =\lim _{j \rightarrow+\infty} \int_{-i T}^{i T}\left(\left|x_{k_{j}}(t)\right|^{2}+\left|x_{k_{j}}^{\prime}(t)\right|^{2}\right) d t \\
& \leq \lim _{j \rightarrow+\infty} \int_{-k_{j} T}^{k_{j} T}\left(\left|x_{k_{j}}(t)\right|^{2}+\left|x_{k_{j}}^{\prime}(t)\right|^{2}\right) d t \\
& \leq D_{1}^{2}+D_{2}^{2} .
\end{aligned}
$$

Let $i \rightarrow+\infty$, then

$$
\int_{-\infty}^{\infty}\left(\left|x_{0}(t)\right|^{2}+\left|x_{0}^{\prime}(t)\right|^{2}\right) d t=\lim _{j \rightarrow+\infty} \int_{-i T}^{i T}\left(\left|x_{0}(t)\right|^{2}+\left|x_{0}^{\prime}(t)\right|^{2}\right) d t \leq D_{1}^{2}+D_{2}^{2}
$$

which implies

$$
\lim _{r \rightarrow+\infty} \int_{|t| \geq r}\left(\left|x_{0}(t)\right|^{2}+\left|x_{0}^{\prime}(t)\right|^{2}\right) d t=0
$$

Using Lemma 2.2, we have

$$
\begin{aligned}
\left|x_{0}(t)\right| & \leq(2 T)^{-\frac{1}{2}}\left(\int_{t-T}^{t+T}\left|x_{0}(s)\right|^{2} d t\right)^{\frac{1}{2}}+T(2 T)^{-\frac{1}{2}}\left(\int_{t-T}^{t+T}\left|x_{0}^{\prime}(s)\right|^{2} d t\right)^{\frac{1}{2}} \\
& \leq 2\left((2 T)^{-\frac{1}{2}}+T(2 T)^{-\frac{1}{2}}\right)\left(\int_{t-k T}^{t+k T}\left(\left|x_{0}(s)\right|^{2}+\left|x_{0}^{\prime}(s)\right|^{2}\right) d t\right)^{\frac{1}{2}} \\
& \rightarrow 0, \quad \text { as }|t| \rightarrow+\infty .
\end{aligned}
$$


Finally, we prove that

$$
x_{0}^{\prime}(t) \rightarrow 0 \quad \text { as }|t| \rightarrow+\infty
$$

From (3.17), we get

$$
\left|x_{0}(t)\right| \leq M_{1}+1, \quad\left|x_{0}^{\prime}(t)\right| \leq \frac{1-\varepsilon_{1}}{\sqrt{2 \varepsilon_{1}-\varepsilon_{1}^{2}}}, \quad \text { for } t \in \mathbb{R}
$$

Then we have

$$
\left|\left(\frac{x_{0}^{\prime}(t)}{\sqrt{1+\left(x_{0}^{\prime}(t)\right)^{2}}}\right)^{\prime}\right| \leq\left|f\left(x_{0}^{\prime}(t)\right)\right|+\left|g\left(x_{0}(t)\right)\right|+|e(t)| \leq M_{5},
$$

where $M_{5}$ is a positive constant.

If (3.21) does not hold, then there exists a sequence $t_{k}$ satisfying

$$
\left|t_{1}\right|<\left|t_{2}\right|<\left|t_{3}\right|<\cdots \quad \text { with }\left|t_{k+1}\right|-\left|t_{k}\right|>1, k=1,2, \ldots
$$

and $\varepsilon_{2} \in\left(0, \frac{1}{4}\right)$ such that

$$
\left|x_{0}^{\prime}\left(t_{k}\right)\right| \geq \frac{2 \varepsilon_{2}}{\sqrt{1-\left(2 \varepsilon_{2}\right)^{2}}}, \quad k=1,2, \ldots
$$

Hence we have, for $t \in\left[t_{k}, t_{k}+\frac{\varepsilon_{2}}{1+M_{5}}\right]$,

$$
\begin{aligned}
\left|x_{0}^{\prime}(t)\right| & \geq\left|\frac{x_{0}^{\prime}(t)}{\sqrt{1+\left(x_{0}^{\prime}(t)\right)^{2}}}\right| \\
& =\left|\frac{x_{0}^{\prime}\left(t_{k}\right)}{\sqrt{1+\left(x_{0}^{\prime}\left(t_{k}\right)\right)^{2}}}+\int_{t_{k}}^{t}\left(\frac{x_{0}^{\prime}(s)}{\sqrt{1+\left(x_{0}^{\prime}(s)\right)^{2}}}\right)^{\prime} d s\right| \\
& \geq\left|\frac{x_{0}^{\prime}\left(t_{k}\right)}{\sqrt{1+\left(x_{0}^{\prime}\left(t_{k}\right)\right)^{2}}}\right|-\int_{t_{k}}^{t}\left|\left(\frac{x_{0}^{\prime}(s)}{\sqrt{1+\left(x_{0}^{\prime}(s)\right)^{2}}}\right)^{\prime}\right| d s \\
& \geq 2 \varepsilon_{2}-\frac{M_{5}}{1+M_{5}} \cdot \varepsilon_{2} \\
& >\varepsilon_{2} .
\end{aligned}
$$

Therefore,

$$
\int_{-\infty}^{+\infty}\left|x_{0}^{\prime}(t)\right|^{2} d t \geq \sum_{k=1}^{\infty} \int_{t_{k}}^{t_{k}+\frac{\varepsilon_{2}}{1+M_{5}}}\left|x_{0}^{\prime}(t)\right|^{2} d t=\infty
$$

which is a contradiction. Then (3.21) holds.

By $\left(\mathrm{H}_{3}\right)$, we have $e \neq 0$. Thus it follows from $f(0)=0$ and $g(0)=0$ that $x_{0}$ is nontrivial. 


\section{An example}

In this section, we shall construct an example to show the applications of Theorem 3.2.

Example 4.1 Let $f(x)=\frac{x^{3}+x}{\sqrt{1+x^{4}}}, g(x)=-\frac{x^{5}+x}{\sqrt{1+x^{8}}}$. The prescribed mean curvature Rayleigh equation

$$
\left(\frac{x^{\prime}}{\sqrt{1+x^{\prime 2}}}\right)^{\prime}+f\left(x^{\prime}(t)\right)+g(x(t))=\exp \left\{-t^{2}\right\}
$$

has at least one nontrivial homoclinic solution.

Proof Obviously, one has

$$
\frac{1}{\sqrt{2}} \cdot x^{2} \leq x f(x) \leq 2 x^{2}
$$

and

$$
x g(x) \leq-\frac{1}{\sqrt{2}} \cdot x^{2}
$$

Then $\left(\mathrm{H}_{1}\right)$ and $\left(\mathrm{H}_{2}\right)$ hold. Since

$$
d=\max \left\{\left(\int_{-\infty}^{+\infty}\left(\exp \left\{-t^{2}\right\}\right)^{2} d t\right)^{\frac{1}{2}}, \sup _{t \in \mathbb{R}} \exp \left\{-t^{2}\right\}\right\}=\sqrt[4]{\frac{\pi}{2}}
$$

$\left(\mathrm{H}_{3}\right)$ holds. Therefore, (4.1) has at least one nontrivial homoclinic solution.

\section{Competing interests}

The authors declare that they have no competing interests.

\section{Authors' contributions}

All authors have equally contributed in obtaining new results in this article and also read and approved the final manuscript.

\section{Author details}

${ }^{1}$ Department of Scientific Research Management, Jiujiang University, Jiujiang, 332005, China. ${ }^{2}$ School of Science, Jiujiang University, Jiujiang, 332005, China.

Received: 5 September 2014 Accepted: 17 February 2015 Published online: 05 March 2015

\section{References}

1. Bergner, M: On the Dirichlet problem for the prescribed mean curvature equation over general domains. Differ. Geom. Appl. 27, 335-343 (2009)

2. Rey, O: Heat flow for the equation of surfaces with prescribed mean curvature. Math. Ann. 297, 123-146 (1991)

3. Benevieri, P, do Ó, J, Medeiros, E: Periodic solutions for nonlinear systems with mean curvature-like operators. Nonlinear Anal. 65, 1462-1475 (2006)

4. Benevieri, P, do Ó, J, Medeiros, E: Periodic solutions for nonlinear equations with mean curvature-like operators. Appl. Math. Lett. 20, 484-492 (2007)

5. Li, W, Liu, Z: Exact number of solutions of a prescribed mean curvature equation. J. Math. Anal. Appl. 367(2), 486-498 (2010)

6. Pan, $\mathrm{H}$ : One-dimensional prescribed mean curvature equation with exponential nonlinearity. Nonlinear Anal. 70, 999-1010 (2009)

7. Pan, $\mathrm{H}$, Xing, R: Time maps and exact multiplicity results for one-dimensional prescribed mean curvature equations. II. Nonlinear Anal. TMA 74(11), 3751-3768 (2011)

8. Amster, P, Mariani, MC: The prescribed mean curvature equation for nonparametric surfaces. Nonlinear Anal. 53(4), 1069-1077 (2003) 
9. Bonheure, D, Habets, P, Obersnel, F, Omari, P: Classical and non-classical solutions of a prescribed curvature equation. J. Differ. Equ. 243, 208-237 (2007)

10. Feng, M: Periodic solutions for prescribed mean curvature Liénard equation with a deviating argument. Nonlinear Anal., Real World Appl. 13, 1216-1223 (2012)

11. Ma, R, Jiang, L: Existence of positive solutions of one-dimensional prescribed mean curvature equation. Math. Probl. Eng. 2014, Article ID 610926 (2014)

12. Lu, S, Lu, M: Periodic solutions for a prescribed mean curvature equation with multiple delays. J. Appl. Math. 2014, Article ID 909252 (2014)

13. Li, J, Wang, Z: Periodic solutions for prescribed mean curvature Rayleigh equation with a deviating argument. Adv. Differ. Equ. 2013, Article ID 88 (2013)

14. Li, J, Luo, J, Cai, Y: Existence and uniqueness of anti-periodic solutions for prescribed mean curvature Rayleigh equations. Bound. Value Probl. 2012, Article ID 109 (2012)

15. Liang, Z, Lu, S: Homoclinic solutions for a kind of prescribed mean curvature Duffing-type equation. Adv. Differ. Equ. 2013, Article ID 279 (2013)

16. Li, J, Wang, Z: Erratum: Existence and uniqueness of anti-periodic solutions for prescribed mean curvature Rayleigh equations. Bound. Value Probl. 2014, Article ID 204 (2014)

17. Lu, S: Homoclinic solutions for a class of second order neutral functional differential system. Acta Math. Sci. 33B(5), 1361-1374 (2013)

18. Lu, S: Homoclinic solutions for a class of second-order $p$-Laplacian differential system with delay. Nonlinear Anal., Real World Appl. 12(1), 780-788 (2011)

19. Lu, S: Existence of homoclinic solutions for a class of neutral functional differential equations. Acta Math. Sin. 28 1261-1274 (2012)

20. Lu, S: Homoclinic solutions for a second-order $p$-Laplacian functional differential system with local condition. Adv. Differ. Equ. 2014, Article ID 244 (2014)

21. Gaines, R, Mawhin, J: Coincidence Degree and Nonlinear Differential Equations. Lecture Notes in Mathematics, vol. 568. Springer, Berlin (1977)

22. Tang, $X, X i a o, L$ : Homoclinic solutions for ordinary $p$-Laplacian systems with a coercive potential. Nonlinear Anal. TMA 71, 1124-1322 (2009)

\section{Submit your manuscript to a SpringerOpen ${ }^{\circ}$ journal and benefit from:}

- Convenient online submission

Rigorous peer review

- Immediate publication on acceptance

- Open access: articles freely available online

- High visibility within the field

- Retaining the copyright to your article 